\title{
Spatial and Temporal Behavior of Tourism Flow Based on Tourism Digital Footprint: A Case Study of Jiuzhaigou Scenic Area
}

\author{
Cao Zihong ${ }^{1, a}$ \\ ${ }^{1}$ Department of Hospitality Management, The Sanda University, Pudong New District, Shanghai, China \\ a593893028@qq.com
}

\begin{abstract}
In the Internet era, the network travel notes shared by tourists on tourism-related platforms can provide a large number of open data resources for the study of tourists' space-time behavior, and these data resources provide a reference for the study of regional tourism's space-time behavior. In this paper, 118 travel notes recorded by tourists on Ctrip and Mafengwo after their visit to Jiuzhaigou from October 10, 2016 to October 10, 2018 were collected as the basic data of the paper, and social network analysis methods were applied to study the spatio-temporal behavior characteristics of tourist flow in Jiuzhaigou scenic spot.
\end{abstract}

Keywords: Spatiotemporal behavior of tourism, Online travel diary

\section{INTRODUCTION}

This research attempts to Jiuzhaigou by tourists travel information network, for tourists to visit the specific line, order of entry by visitors to the site and time, on the basis of network data travels, jiuzhaigou tourism flow network built using social network analysis, analysis of jiuzhaigou tourism flow network node centricity, Jiuzhaigou tourism flow behavior characteristics of space and time are obtained[1].This paper attempts to provide reference for the competition and cooperation of scenic spots, scenic spot optimization and suggestions through the study of tourism flow among scenic spots in Jiuzhaigou scenic spot.

\section{THE RESEARCH SCOPE}

Jiuzhaigou World Natural Heritage Site, located in Jiuzhaigou County, Aba Tibetan and Qiang Autonomous Prefecture, Sichuan Province, covers an area of 720 square kilometers. The research scope selected in this study mainly focuses on Shuzhenggou scenery line, Rizegou scenery line and Zechawagou scenery line, three scenery lines are Y-shaped. In this area, the best tourism resources and scenic spots of the whole scenic area are concentrated and distributed, such as Nuo Rilang, Jinghai, Zhenzhutan, Wuhuahai, Xiongmaohai, Jianzhuhai, Yuanshisenlin, Changhai, and Wucaichi, etc., and the tourist service facilities are perfect.

\section{DATA SOURCE AND SCREENING}

Enter the keyword "Jiuzhaigou" into the search interface of Ctrip and Mafengwo, and enter the "travel notes" section in the search results. The search results will be sorted according to "popular" and the number of "likes" and "tops" of travel notes will be referred to[2]. A total of 118 travel notes recorded by tourists on Ctrip and Mafengwo websites after they visited Jiuzhaigou from October 10, 2016 to October 10, 2018 were collected as the basic data of this paper.

The specific screening criteria are as follows: $\square$ The travel notes record the specific travel time, or the travel time can be restored according to the context of the travel notes, and the travel time is between October 10, 2014 and October 10, 2016, otherwise it will not be recorded. $\square$ The content of travel notes involves the travel routes and scenic spots within the scope of the study area, and the travel notes that directly describe the scenic spots are preferred. The content is complete, can restore the tourists in Jiuzhaigou actual scenic spots tour order, if only the list of scenic spots will be removed. A total of 267 travel notes are involved, of which 118 can be used as the data basis. Among them, Mafengwo posted 56 and Ctrip 62. 


\section{ANALYSIS}

\subsection{The temporal characteristics of tourism flow in Jiuzhaigou are seasonal}

The tourist flow in Jiuzhaigou scenic spot has strong time fluctuation and seasonality, and the data show an increase in fluctuation. There are obviously three peaks, which appear in June, October and December respectively, and the peak in October is significantly higher than the other two peaks. In the time change of a year, the peak of tourism is concentrated in August to October, and the tourist flow in three months is $48.15 \%$, accounting for almost half of the whole year. Jiuzhaigou scenic spot has a large number of unique natural tourism resources and cultural tourism resources mainly in Tibetan villages. These water resources are obviously affected by seasonal changes. In autumn, the water flows abundantly, reflecting with the surrounding red maple leaves and colorful leaves, which are popular with tourists. The winter and spring are long without rain, and the waterfall is frozen, although it also has a different taste, but slightly inferior to the late autumn. Second, in winter, for safety reasons, the Yuanshisenlin forest will be closed off, and the shuttle bus in the scenic spot will only go to the arrow bamboo sea scenic spot.

\subsection{Analysis of tourist attraction preference in Jiuzhaigou}

The frequency and proportion of each scenic spot in Jiuzhaigou tourist route are calculated through the data of online travel notes, so as to analyze the preference of Jiuzhaigou tourists for different scenic spots. A total of 20 scenic spots were collected in this study, among which 7 scenic spots appeared more than 7.0\% frequently, accounting for $54.3 \%$ of all scenic spots. These scenic spots almost represent the essence of Jiuzhaigou scenic spot tourism. In addition, there are 8 scenic spots with a frequency ratio greater than 3.0\% and less than $7.0 \%$, accounting for $38.8 \%$ of all scenic spots, and the other 5 scenic spots with a frequency ratio less than $3.0 \%$. The whole distribution is relatively stable, and the scenic spots that tourists especially prefer are not very prominent.

\subsection{Construction of tourism flow network structure in Jiuzhaigou}

The nodes mentioned in tourists' online travel notes are sorted into an assignment matrix of $\mathrm{N} * \mathrm{~N}$ according to their tour order. Where $\mathrm{N}$ is the number of tourism nodes mentioned in the travel notes of Jiuzhaigou, rows and columns represent tourism nodes, and assignment matrix elements $(A, B)$ represent the number of one-way trips of tourists from tourism node A to tourism node B. All flow direction relationships are summed up successively to form the assignment matrix of social network relations of Jiuzhaigou tourist attractions, with A scale of "20*20". A document in the format ".\#\# "is outputted by UCINET for NetDraw to build the Tourism Network Structure Map[3].

On the basis of the social network assignment matrix of Jiuzhaigou tourist attractions, the binarization processing is carried out, and the appropriate breakpoint value can not only highlight the key points, but also not ignore the important nodes. If the flow between two tourism nodes is greater than the breakpoint value, it is denoted as "1" in the dichotomy matrix. Otherwise, it is marked as "0". After many attempts and comparisons, "2" was finally selected as the breakpoint value, and 20 tourism nodes were retained. Through the document output by UCINET as "Gt2.\#\#H",centrality visualization analysis was carried out in NetDraw, and the tourism flow network structure diagram of Jiuzhaigou was constructed[4]. The size of the circle represents the centrality of the scenic spot. The larger the circle, the greater the centrality of the scenic spot, and vice versa. The thickness of the line segment represents the size of the tourist flow between two scenic spots. The thicker the line segment, the greater the tourist flow. Through the connection of the thicker line segment, we can see the tourist route that most tourists will choose[5]. As can be seen from Figure 1, nodes with a higher degree of center mainly include Nuorilang, Wucaichi, Xiniuhai, Jianzhuhai, Changhai, Shuzheng qunhai and Jinghai. These scenic spots themselves are also closely related. The greater the center degree, the closer the connection with other scenic spots is. 


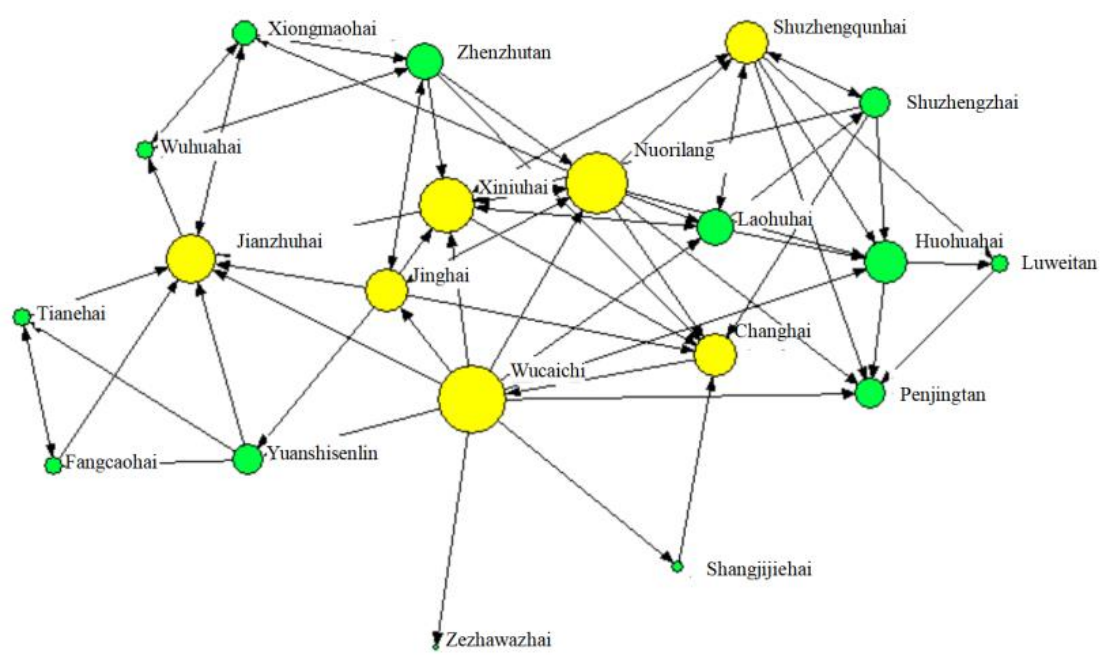

Figure 1 Tourism flow network structure of Jiuzhaigou.

\subsection{Analysis results of tourism flow network structure in Jiuzhaigou}

In the 20 nodes of Jiuzhaigou scenic spot selected, from the perspective of degree centrality, each tourism node has agglomeration and radiation relation with 7.3 other tourism nodes on average. From the perspective of standard deviation, the indexes of absolute centrality and relative centrality of degree centrality are 3.116 and 16.400 , respectively, which have certain differences. In the tourism network of Jiuzhaigou, the node with the highest degree centrality index is Nuorilang Waterfall, which has the largest number of connected scenic spots, indicating that Nuorilang has a strong influence and dominance on other scenic spots in the course of tourists' tour.

From the perspective of proximity centrality, the mean values of introversion and extroversion are 26.159 and 43.329 , respectively. The degree of dependence of tourist flows in and out of scenic spots on other scenic spots is different, and introversion is low while extroversion is high. From the standard deviation, inward and outward are 5.576 and 16.728 , respectively, indicating that the distribution of Jiuzhaigou tourism nodes in the tourism network is not very balanced. But in general, most of the sites are closely connected. Several scenic spots, such as Nuorilang, Wucaichi and Xiniuhai, have high internal and external proximity centrality index, indicating that they are likely to appear on the tour itinerary of tourists. However, there was a great difference in the value of introversion and extroversion near centrality of Penjingtan, and the value of introversion near centrality was the largest (introversion 47.50, extroversion 5.00). When it serves as an entry point, it is less dependent on other nodes, which is in line with the situation that the Penjingtan near the goukou is the starting point or the last stop of tourists' trip. Moreover, it is directly connected with the core tourism nodes and is not controlled by other tourism nodes to a large extent.From the perspective of centrality, in the tourism flow network, each tourism node acts as a transfer station for 20.85 times on average. Among the standard deviation indicators of centrality, the standard deviation of the centrality of intermediation is the largest, which is 24.415 , indicating that there is a great difference in the neutral intermediation of nodes in the tourism network. Some core tourism nodes are outstanding transit stations, and many tourism flows pass through these core nodes. Nuorilang has the largest degree of intermediation centrality, with a value of 96.250 , indicating that most tourist flows go through it to other tourist nodes. Nuorilang, which is located in the center of Jiuzhaigou scenic area, is a very important hub in the whole tourism flow network of Jiuzhaigou.

All of the tourism festival point centrality indices and jiuzhaigou tourism flow network structure, Nuorilang, Wucaichi, Xiuniuhai, Jianzhuhai, Changhai, Shuzhengqunhai, Jinghai 7 spots centricity is very high, the degree of emphasis in tourism network, has the very strong aggregation and radiation function. But at the same time, combining with the tourists' preferences of jiuzhaigou scenic spot, found in jiuzhaigou tourism flow center node in the network structure and the actual popular with tourists in the tourism node there is a discrepancy, such as on the long centricity's highest waterfall is not the most popular with tourists attractions, in long beach, the most popular with tourists, but not in the mind of the highest node.

\section{CONCLUSION}

(1) Tourists have an obvious preference for scenic spots in Jiuzhaigou, which is manifested as lakes and waterfalls rich in natural water resources and famous locations for filming films and TV dramas, while scenic spots with strong seasonal patterns will reduce their preference for Jiuzhaigou. Changhai, Zhenzhutan, Wucaichi and Wuhuahai are the most popular scenic spots for tourists. 
(2) The tourism flow network has a high density and more overall tourist routes. Nuorilang, Wucaichi, Xiniuhai, Jianzhuhai, Changhai, Shuzhengqunhai and Jinghai have a high node centrality, and play a core role in the tourism flow network of Jiuzhaigou. The 20 scenic spots in Jiuzhaigou can be divided into four types by combining the center degree and preference degree. Among the 20 scenic spots in Jiuzhaigou, Changhai, Wucaichi, Nuorilang and Jianzhuhai are the most popular tourist nodes located in the center of the network. Finally, the transportation system, tourist routes and infrastructure of Jiuzhaigou Scenic Area can be optimized, so as to meet the tourism needs of tourists and improve their tourism experience while protecting their safety.

\section{REFERENCES}

[1] Fabien G, Josep B, Francesco C, et al. (200 8)Digital Footprinting: Uncovering Tourists w ith User-Generated Content. J. Pervasive Com puting, IEEE, 7(4) : 36-43.

[2] Xinyi DAI. (2016)Behavior Characteristics and Spatial Characteristics of Bicycle Riding Based on the Content Analysis of Network Text.Inter national Journal of Simulation -- Systems, Scie nce \& Technology.17: p1-5, 5p.

[3] Andrea V, Liang L, Assaf B, et al. (2009)A ho listic framework for the study of urban traces and the profiling of urban processes and dyna mics. J. Intelligent Transportation Systems, ITS C '09.12th International IEEE Conference on In telligent Transportation Systems:1- 6 .

[4] Girardin F, Fiore F D, Ratti C, et al. (2008) Leveraging explicitly disclosed location informat ion to understand tourist dynamics: A case stud y. Journal of Location Based Services, 2(1): 4 $1-56$.

[5] Li, Yang., Zhang, Lei. (2020)Ecological efficien cy management of tourism scenic spots based o $\mathrm{n}$ carbon footprint analysis. International Journal o f Low Carbon Technologies. 15:550-554. 